\title{
A Framework to Guide ICT Solution for Language Barrier in South African Healthcare
}

Phathutshedzo Makovhololo, Cape Peninsula University of Technology, South Africa

Tiko Iyamu, Cape Peninsula University of Technology, South Africa

\begin{abstract}
In recent years, healthcare service providers have increasingly employed mobile systems in delivering services. However, the nature of the diversities in tribes and languages within many developing countries enhance the difficulty of delivering or receiving the services which are provided by many healthcare organisations. This is so because the English language is the primary medium of communication, although many healthcare recipients are not conversant in English as they cannot speak English fluently or understand it coherently. The objective of the study was to understand the impact which language barrier have on healthcare services. The interpretivist approach was employed. The case study approach was applied. Qualitative data were collected using semi-structured technique. The analysis of the data was guided by two theories, actor network theory and diffusion of innovation. From the findings, a framework was developed, which is intended to guide a solution that can be provided to address the barrier in the South African healthcare environment.
\end{abstract}

\section{KEYWORDS}

Information Technology, Language Semantics, Actor Network Theory, Diffusion Of Innovation, South Africa

\section{INTRODUCTION}

The need for Information and Communication Technology (ICT) has increased monumentally in recent years as nearly everyone has witnessed a vast expansion in the prevalence of mobile device (Brown, 2018). ICT solutions are used to facilitate communication, information processing, storage and management through electronic means (Saleem, Flanagan, Wilck, Demetriades, \& Doebbeling, 2013, p. 49). Also, ICT is considered as the key to enhancing and improving communication among healthcare providers, especially for patients with chronic conditions (Barr, Vania, Randall, \& Mulvale, 2017). According to Yagos, Olok and Ovuga (2017), services such as e-Referral, teleconsultation, electronic medical records, and the utilisation of mobile phones indicate substantial benefits to health workers in rural and remote areas. Thus, ICT can be used to promote and improve the quality of healthcare and enhance the education of both health professionals and patients (Rouleau, Gagnon, \& Côté, 2015).

However, healthcare service delivery systems have faced problems (Ball, Bertone, Carrazza, Deans, Del Debbio, Forte, Guffanti, Hartland, Latorre, Rojo, \& Ubiali, 2015). Some of the problems include usability, information security (Zakaria, Affendi, \& Zakaria, 2010), as well as the translation of languages. According to Nemutanzhela and Iyamu (2016), the diverse nature of the tribes and languages within African countries makes it difficult to deliver or receive the provisional services of

This article, originally published under IGI Global's copyright on January 24, 2020 will proceed with publication as an Open Access article starting on January 18, 2021 in the gold Open Access journal, Journal of Cases on Information Technology (converted to gold Open Access January 1, 2021), and will be distributed under the terms of the Creative Commons Attribution License (http://creativecommons.org/licenses/ by/4.0/) which permits unrestricted use, distribution, and production in any medium, provided the author of the original work and original publication source are properly credited. 
healthcare organisations, by using ICT solutions. Some of these challenges can be attributed to the fact that many of the ICT solutions for healthcare are rarely fulfilled as they are frequently developed without valuable input from patients (Vermeulen, Portengen, Silverman, Garshick, \& Steenland, 2014).

Due to the numerous languages, other than English, in South Africa, there is a clear need for translation so that both the healthcare service providers and receivers can benefit. Dong and Cremers (2014) assert that translation of languages is not easy or straightforward as is popularly acclaimed. This is primarily because of the semantics in some of the languages. Semantics of language is referred to in this study as the meanings of words and sentences (Carston, 2013), which often have impact on the interactions that occur between healthcare service providers and the recipients.

Based on the challenges stated above, the objective of the study was to understand the impact which language barriers have on healthcare services. The article is divided into six main sections. The first section presents introduction, which is followed by the literature review. The third section covers the methodology that was applied in the study. In the fourth and fifth sections, data analysis and findings are presented, respectively. Finally, a conclusion of the study is drawn.

\section{LITERATURE REVIEW}

The advancement in ICT solutions has over the years increasingly managed to improve an efficient distribution of healthcare services to people in rural areas. According to Iftikhar., Ishaq, Ahmad, \& Fatima (2010), health informatics play a vital role in the integration of ICT solutions for healthcare purposes. This includes data storage and retrieval (Rodger, 2015). Despite advancements in ICT solutions, language barriers have been shown to affect the quality of healthcare received by limited English proficiency patients. "Language is a mirror of the mind in a deep and significant sense. It is a product of human intelligence" (Chomsky, 1975 p. 4; Idalovichi, 2014). In late 1999, the Institute of Medicine highlighted the effects of language barriers in its report on medical errors and patient safety (Kohn, Corrigan, \& Donaldson, 1999). Error rates were higher when physicians and patients spoke different languages (Gandhi, Burstin, Cook, Puopolo, Haas, Brennan, \& Bates, 1998). Woloshin, Bickell, Schwartz, Gany, \& Welch (1995) associate language barriers and inaccurate with errors in medical history and misdiagnosing of medical conditions.

Research shows that patient satisfaction increases when interpreter services were employed as this reduced language barriers (Baker, Hayes, \& Fortier, 1998). Language skills are not only about listening, speaking, reading, or writing, but also include translation. According to Anari and Sanjarani (2016 p. 145), "translation, involving the transposition of thoughts expressed in one language by one social group into the appropriate expression of another group, entails a process of cultural de-coding, re-coding and en-coding". From the other side of the coin, Gany et al. (2007) explain that translation of medical interaction by unqualified interpreters is prone to omissions, additions, substitutions, volunteered opinions, and semantic errors that can seriously distort care with potential drastic results. Basically, communicative translation emphasises the swift passage of messages (Munday, 2016).

The South African healthcare environment is currently changing, being transformed to provide new benefits and fulfil opportunities through technological innovations, such as mobile technology (Nemutanzhela \& Iyamu, 2016). However, these changes usher in both challenges and potential barriers. Overcoming the use of various languages within the healthcare system is one of the critical barriers (Naidoo, 2014) affecting the well-being of millions of people living in South Africa. Of the approximately 54 million people in this country, only 4.9 million people are first-language English-speakers, while the rest speak a variety of other languages such isiZulu, isiXhosa, Sepedi, Tshivenda and Xitsonga as their first languages. For many, their first language is their only language of communication. Consequently, the present healthcare service delivery system is struggling to handle this diversity (Burns, 2012). According to Ayong and Atanga (2017), the issue of healthcare professionals having to care for patients of different cultural backgrounds and language barriers poses numerous critical challenges to the healthcare systems. This necessitates redress through an advancement, such as a mobile system. 


\section{RESEARCH METHODOLOGY}

Qualitative data were collected using the semi-structured interview technique. This was primarily because the qualitative method allows for individual subjective views and association of meanings (Grove, Burns, \& Gray, 2014). Thus, the method was most suitable because the research aimed to gather views about the use of various language in providing and receiving healthcare services, from individuals and groups.

The case study approach was applied in the study for the purposes of gaining better understanding of a real-life situation, by following the Yin's (2013) approach. Mukhethwa Hospital (MH) was used as a case in the study. This was because the hospital serves the most diverse (see Table 1 community in South Africa, in terms of spoken languages. The hospital is a public hospital in Atteridgeville, Pretoria, Gauteng province of South Africa. It was established in 1973 to provide service to the black populace, serves estimate of 2 million people. According to the 2011 census, the most commonly spoken language in the area is Northern Sotho (Sepedi), followed by Tswana and Sesotho, as shown in Table 1.

The interviews were conducted in the language of the respondent choice. The interviewees were recorded. Participants were selected based on criteria such as: a patient must have visited the hospital for at least more than twice in a year; a healthcare personnel mush have been working in the facility for at least two years; and the participant must be interested in sharing personal experience. Example of the interview questions are: What are the factors of influence in spoken languages, and how can its semantics be translated from local dialect to English by using mobile systems, in order to improve healthcare services in South Africa? How does the translation of language semantics impact the services that healthcare organisations provide to the communities in South Africa?

One-on-one interviews were carried on face-to-face basis (Farooq \& de Villiers, 2017) with the participants, which allowed the participants to share their views without shyness and influence by a third party. Participants were selected from the five most spoken languages, as shown in Table 1, which make up $84.53 \%$ of the total population served by the hospital at the time of this study. As shown in Table 2, a total of 27 people were interviewed at the point of saturation, which means that

Table 1. Atteridgeville demographics (Census, 2011)

\begin{tabular}{|l|l|l|}
\hline Language & People & Percentage \\
\hline Sepedi & 26236 & $41.38 \%$ \\
\hline Setswana & 10566 & $16.67 \%$ \\
\hline Sesotho & 7823 & $12.34 \%$ \\
\hline isiZulu & 4550 & $7.18 \%$ \\
\hline Xitsonga & 4412 & $6.96 \%$ \\
\hline Tshivenda & 2299 & $3.63 \%$ \\
\hline isiNdebele & 2149 & $3.39 \%$ \\
\hline English & 1632 & $2.57 \%$ \\
\hline isiXhosa & 1204 & $1.90 \%$ \\
\hline SiSwati & 896 & $1.41 \%$ \\
\hline Other & 803 & $1.27 \%$ \\
\hline Afrikaans & 438 & $0.69 \%$ \\
\hline Sign language & 390 & $0.62 \%$ \\
\hline Not applicable & 1027 & \\
\hline
\end{tabular}


new information was not forthcoming. The qualitative method is not about statistical procedures or other means of quantification, instead (Baškarada, 2014). For the purposes of analysis, the 27 interviewees were labelled MH01 to MH27. The participants were coded, and a referencing format was adhered to as follows: case name, participant code, page number and line number. For example, TC01, 4:15-17 indicates participant number 1, page 4 of the interview transcript, and line numbers 15 to 17 of the document.

The moments of translation from the Actor Network Theory (ANT) (Callon, 1986) and the Diffusion of Innovation (DOI) from the perspective of Innovation-Decision Process (Rogers, 2003) were used as lenses to guide the analysis of data, which was conducted by using the hermeneutic approach.

ANT consists of actor and network whereby neither of them is independent of the other (Callon, 1986). ANT focuses on the transition of networks (Iyamu, 2015). Translation is a process of negotiation through four different moments: problematisation, interessement, enrolment, and mobilisation from which actors realise their actor-worlds (Callon, 1986). In problematisation, an initiative is articulated for a possible solution (Iyamu, 2015). Interessement is the translation process where aligned interest is established. In the enrolment stage, the focal actor attempts to define roles for other actors (Tatnall, 2012). Finally, is the moment of mobilisation of allies to ensure actors representatives and inclusiveness through persuasion by other actors (Walsham, 1997).

DOI is the process by which an idea, practice, or object that is perceived as new by an individual or other is communicated through certain channels over a period of time within a social system (Rogers, 2003). Innovation is defined as "an idea, practice or object that is perceived as new, whether or not it is objectively new as measured by the lapse of time since its first use or discovery" (Rogers, 2003 p. 12). Diffusion focuses on awareness and knowledge about innovation, and examines attitude change, decision making, and implementation of the innovation (Aizstrauta, Ginters, \& Eroles, 2015).

\section{DATA ANALYSIS AND FINDINGS}

\section{Actor-Network Theory}

As depicted in Figure 1, both human and non-human actors were involved in the process of providing healthcare services. In MH, human actors were categorised into three groups: healthcare practitioners, administrators, and IT personnel. The health practitioners included doctors (general practitioners, gynaecologists, and neurosurgeons), nurses, and pharmacists. According to one of the nurse participants, "we don't only rely on the nurses alone to translate, any staff members for example, clerks, secretary, pharmacist and data capturers also assists" (MH01, 3:79-81). The administrative personnel consist of managers, clerical assistants, human resources, and IT personnel (IT manager, technicians, and database administrators). The non-human actors at MH were identified using two categories: technical and non-technical actors. The non-technical actors included the process, medications, and

Table 2. Mukhethwa workforce demographics

\begin{tabular}{|l|l|l|l|}
\hline \multicolumn{1}{|c|}{ Employee } & \multicolumn{1}{|c|}{ Strength } & \multicolumn{1}{c|}{ Interviewee } & \multicolumn{1}{c|}{ Language } \\
\hline Administrators & 1653 & 5 & Sesotho (x3); Setswana (x1); isiZulu (x1) \\
\hline Doctors & 139 & 7 & Sepedi (x3); Setswana (x2); isiZulu (x1); Sesotho (x1) \\
\hline Nurses & 1236 & 6 & Setswana (x2); Sepedi (x2); Xitsonga (x1); isiZulu (x1) \\
\hline Pharmacists & 24 & 3 & isiZulu (x2); Sepedi (x1) \\
\hline IT Personnel & 38 & 6 & Sepedi (x3); Xitsonga (x2); Sesotho (x1) \\
\hline Total & & 27 & \\
\hline
\end{tabular}




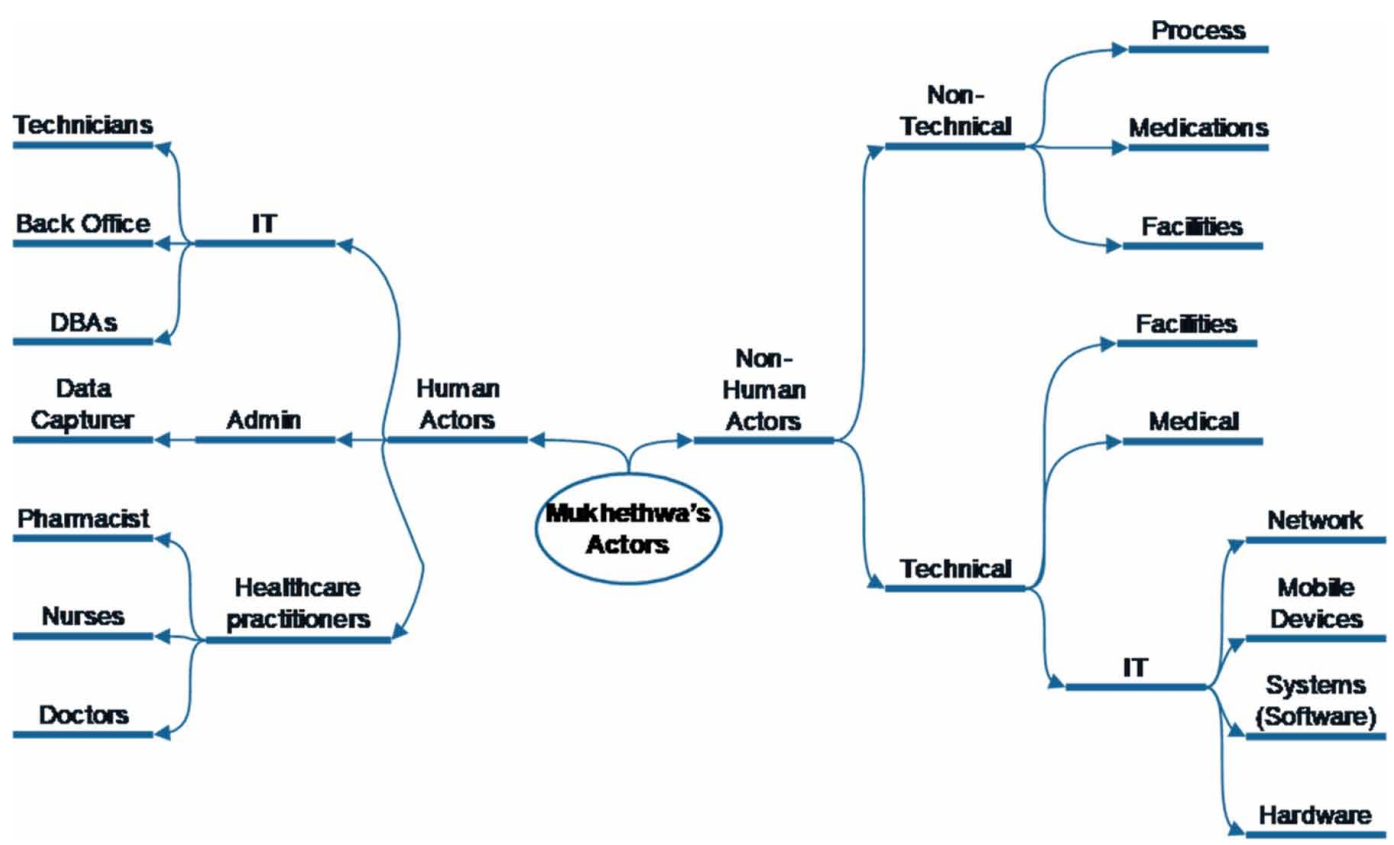

facilities, while the technical actors included the facilities, medical, and information technologies. Together, the actors contributed to the services from various perspectives, such IT, administration and health practice. The non-human consisted of technical and non-technical actors.

\section{Networks}

There were different networks of people, divided into two main groups: technical and non-technical. In this study, technical refers to healthcare practitioners, admin, IT, and patients, while non-technical includes the hospital, systems used, and spoken languages. Within these networks, sub networks existed and replicated themselves, which ANT refers to as heterogeneity (Callon, 1986). In ANT, an actor can join more than one network simultaneously, manifesting itself differently in each network (Iyamu \& Tatnall, 2009).

There was networks formed around areas of specialisations, such as doctors, nurses, pharmacists, administrators, and IT personnel. The challenge for some of the groups is when patient speaks only home language which the practitioners do not understand. According to MH018 (3:86-88) "If we encounter languages issues during consultation with a patient, we are able to resolve that through different staff members that we have irrespective of their occupation, we normally call on our colleagues". One of the nurses explained: "however, at the hospital even though media of communication is English, we have healthcare practitioners who are multilingual, they speak languages, such as Zulu, Sotho, Tsonga, and Northern Sotho" (MH11, 1:18-20). Besides communicating verbally, some members of the networks also used technologies, such as mobile phones and social media services, to communicate and monitor a patient's health while at home. "We have introduced the use of medical devices for patients' self-measurements at home, which can be accessed via cellular phone or laptops or any kind of devices that can take the application" (MH02, 5:148-150).

There were four main categories of patients: (1) patients for general consultations; (2) regular check-up; (3) renewal of prescriptions and collection of medications; and (4) patients diagnosed with a chronic condition. These group of patients come from different designated areas, upon which they unconsciously formed networks influenced by ethnicities. For instance, MH05 (21: 638) pointed out 
that "we have patients who stays at squatter camps (remote areas)". The actors in those networks spoke various languages, such as Tshivenda, Xitsonga, Isizulu, Sesotho, Sepedi and Xhosa. The actors relied on their individual languages to access medical information and receive medications. However, these actors had to interact with healthcare practitioners, such as doctors, nurses, and pharmacists, who speak different languages.

\section{Moments of Translation: Problematisation}

Actors problematised healthcare services, from both front desk and IT support viewpoints, depending on their roles and responsibilities. One IT technician (MH07, 27:844-845) said that "as IT we are responsible to provide users (nurse, doctors, pharmacist and data capturers) with working laptops". In providing support to the healthcare practitioners, communication challenges are experienced. "It's difficult for me to support healthcare practitioners on site as I can only speak English and you find that they want to explain the problem using their home languages" (MH019, 34:1100-1102).

Many of the healthcare practitioners' emphasised the need for language translators in providing healthcare services to the needing. "Language barriers are a serious problem in our hospital, we have lot of foreign patients" (MH01, 1:22-23). However, some of the participants argued that it is not just the language, but the semantics, especially when it comes to medical terms: "The direct translation alone doesn't necessarily give meaning to words, even if you borrow it in English" (MH02, 6:183-184).

South Africa has eleven official languages, which sometimes present serious challenges for a healthcare worker when communicating with patients from different parts of the country. "Even for us South Africans, we don't know all the eleven official languages" (MH05, 22:670-674). This presents a seemingly insurmountable challenge for communication, especially when the patient is a foreigner. Some of these patients who are linguistic minority migrants end up relying on sign languages during their healthcare encounters or rely on the availability and accuracy of an interpreter. According to MH03 (12:370-371), for instance, "we had a scenario where a patient, an Angolan citizen came for surgical operation, and she came with her own interpreter". The challenge with interpreters is that some of them do not understand one of the languages perfectly enough to give accurate meaning, when it comes to healthcare matters, which sometimes misinform or mislead the practitioners. Participant (MH06, 23:725-727) said that "translators don't have to be healthcare practitioner, but need to be fluent in the spoken language of the patients".

During this translation, some of the healthcare practitioners felt that the patients were not free, and they think this might have compromised the information they provided to them. One of the doctors (MH06, 25:791-793) asserted that "We work with nurses, if a patient doesn't understand what you are saying, simply call a nurse who can translate for you". When you communicate with the patient in a language they understand, you form a relationship with them; they become comfortable and can express themselves. Another doctor asserted, "I am lucky because I can communicate with most of my patients in their own languages" (MH08, 23:718-72).

\section{Moments of Translation: Interessement}

The relationship between the healthcare practitioners and their patients was defined by the types of interactions, communication, and association they had. For some of the healthcare practitioners in $\mathrm{MH}$, interests were influenced by different factors; for others, it was because they dealt with patients directly. The interest of $\mathrm{MH}$ was based on delivering the best healthcare services to all who care, irrespective of the language that they speak or understand. Thus, one of the participants (MH01, $2: 57)$ expressed that "as a nurse I must be able to communicate with my patients". This is to ensure proper understanding in providing services. Also, an understanding between practitioners and patients eradicate the use of interpreter, which infringes on privacy.

Different actors were involved in, and influenced, by the relationships between healthcare practitioners and patients. The actors were healthcare practitioners, patients, patients' relatives, associates, support groups, and the systems that were used for communicating and sharing information. 
"We communicate in eleven South African official languages. But we also have patients who are from outside the country, such as from Zimbabwe and Pakistan" (MH05, 18:552-553). Some of the healthcare practitioners had both direct and indirect relationships with some patients. Also, some practitioners were able to communicate with the patients through the help of interpreters. The healthcare practitioners must be able to accommodate all the patients, irrespective of the language they speak. According to one of the head nurses (MH05, 18:560-561, "there is always an interpreter on standby at the hospital who can speak foreign languages".

For these reasons, most of the healthcare practitioners were interested in speaking the patients' language so that they can create a solid relationship while also making patients comfortable: "I communicate in the patients' language so that I can get the real story" (MH06, 23:725-726). However, those who could not understand the language or were unable to communicate with the patients were not discouraged, but south alternatives. According to one of the participants, MH01 (1:50-51), "it was suggestion for nurses to get trained on linguistic including sign language".

\section{Moments of Translation: Enrollment}

At this stage, the actors accept the roles and responsibilities that were proposed or assigned to them by the focal actor, which were their line managers. It is within this frame that most of the healthcare practitioners, such as doctors and nurses, carry out their tasks. Not all the healthcare practitioners who were interested in the healthcare services participate in all the healthcare activities on their own, a situation attributable to different factors which were either conscious or unconscious. This was attributed to language barrier. Both the healthcare workers and patients take these issues seriously in terms of attempting to find a pragmatic solution to these barriers. By doing so, they confirm and enrol into the duties imposed on them in ensuring healthcare services are delivered, and the patient has received the best possible treatment. Thus, both practitioners and patients employ the service of interpreter when necessary (MH03, 12:369-370). Although the use of interpreter did not necessarily address the challenge in that some of the interpreters struggle in translating some semantics. "I could see that the interpreter was not comfortable, and her explanation was short and little. For example, she explained a long paragraph of about 182 words in two sentences" (MH04, 12:370-373).

Families members or friends of patients also assisted in translating conversation between healthcare practitioners and patients. This helps to improve understanding between the parties. "We go to an extent of calling the spouses of patients who are challenged with English language" (MH01, 2:33-35). More so, another participant (MH03, 12:357-358) asserted that "some people come with their kids who speaks better English to translate for them". Another challenge was the role of culture, which plays a big role in the enrolment of actors in providing and receiving healthcare in $\mathrm{MH}$. For example, some female patients refused to allow the male health practitioners to treat them. A male nurse participant MH05 (22:677-679) expresses himself: "culture plays a big hindrance to what we have to do, I once had a woman patient who refused to be assisted by me, because I am a male".

Additionally, some healthcare practitioners made use of visuals, such as pictures on walls or from books or off the internet, to attempt to communicate with the patients at the level that allows them to understand each other. "In case of communication barriers with my patients, I use a computer to show them graphic images" (MH03, 11:345-356). The participant further explained that "this assisted with communication barriers as they nod or shake their heads if they agree or disagree" (MH03, 12:349-351). Furthermore, some patients were even willing to try to speak in the healthcare practitioner's spoken language in order to enhance the possibility of communication. According to one of the doctor participants, MH06 (25:781-783), "I have assisted patients who spoke in English instead of their home languages to accommodate me, because they knew I couldn't speak their languages".

\section{Moments of Translation: Mobilisation}

Mobilisation is the fourth and final stage of translation. In mobilisation, the focal actor needs to continually convince the actors that their interests are still the same. For example, different 
spokespersons were established with different interests. Some of the actors participated as interpreters between the practitioners and the patients, making it their task that both actors can understand each other. This was influenced by the relationship they have with either the patient or healthcare practitioner, or the interest they developed in the health of the patients. Other healthcare practitioners, hospital interpreters, and family members of the patients made it their own responsibility to eliminate communication barriers.

In MH, different spokespersons were established according to specific circumstance. For those patients who are from outside South Africa and unable to speak the language spoken by the healthcare practitioners, they had different spokespersons available such as interpreters, family, and even health practitioners themselves acting as spokespersons, while those in chronic medical conditions had buddies and themselves in this regard. For instance, MH22 (2:34) indicated that "we got to an extent of even calling the husband of the patient to come and translate for us".

Healthcare workers cannot assist a patient if they do not understand the problem or cannot communicate in a language that they both understand. Both the healthcare workers and patients take these issues seriously. As result, they search for a solution to this barrier. By doing so, they were both confirming and enrolling into the duties imposed on them to make sure that at the end of the day the services are delivered and the patient receives good service. This problem goes beyond just language translation: even though some of the participants agreed that the issue concerns language barriers, most of them admitted that the most substantial challenge is with the semantics of languages that are used to communicate at the hospital. Others talk mostly around the translation of medical terms to their language being a big challenge.

\section{Diffusion of Innovation}

The second part of the objective was to examine how technological solutions can be diffused in private hospitals, using mobile devices (i) to address the challenges of language translation for healthcare service purposes, and (ii) to translate the semantics in the languages. The stages of a decision process, from the perspective of the DOI theory (Rogers, 2003), was employed in the analysis of the data. The analysis begins and ends with the knowledge and confirmation components, respectively.

\section{Innovation Decision Process: Knowledge}

Healthcare personnel in Mukhethwa were no different from other health professionals, which require knowledge in carrying out their services. According to Scott and McGuire (2017), knowledge is the initial awareness potential users have about an innovation, as well as the understanding of how the innovation operates. According to MH03 (11:345-347), "I sometimes found myself having to use visuals graphs, images and so forth, to try to explain to my patients what am trying to say". Some of the practices that are executed were based on knowledge gained from experience of over a period of time. Such knowledge covers both cultural practices and technological know-how. For example, at the time of this study, the hospital received a patient of Pakistani origin who was critically ill and could not communicate. The healthcare personnel who were on duty on the day were not knowledgeable of the fact that female patients from Pakistan do not accept care from male practitioners. As a result, the patient fell into a state of depression when she discovered that male healthcare personnel attended to her, which was yet another critical condition. This type of situation explains the critical influence of knowledge within the healthcare environment.

Mobile applications were used for communication between some practitioners and patients. However, many of the patients were not knowledgeable about the mobile applications. Even those who knew about the applications, struggled to use them. Participant MH05 (6:89-90) indicates an instance, that the applications were not really used "because most of the times the patients don't respond to our messages" (MH05, 6:89-90). It is not that everyone is technologically disadvantaged. There are other reasons why some people do not use the applications, which we need to be knowledgeable about. 
Many of the healthcare personnel focused on improving communication between themselves, their care teams, and their patients. With regards to healthcare personnel, they engage with each other using different languages. The challenge that some of the healthcare practitioners were experiencing had to do with language lingo. The community around the Soshaguve township has their own way of speaking, which they created. The challenge of communication caused by language lingos affected service delivery in the area. During the interaction with a patient, the local language lingo was often used which generated some misunderstanding between the healthcare practitioner and the patients. According to one of the nurse participants, MH03 (10: 312-313), "at Soshanguve, ladies like to use the word isibabo, which to them means itching. The actual meaning of the 'Isibabo' is to discharge". A medical practitioner who does not understand this term might prescript medicine for discharges, which is highly likely to have other health implications for the patients.

\section{Innovation Decision Process: Persuasion}

In $\mathrm{MH}$, when the healthcare practitioners and patients interact there were certain things that led to their being persuaded. In the case of patients, for example, some of the patients were persuaded when they had to get a medications prescription. According to MH08 (31:1000-1002), "Even though pharmacist has an electronic copy of patient's prescription, the legislations allows the patient to have a copy". However, some of the patients feel motivated and persuaded when they realise that the healthcare practitioners can interact in their home language. According to one of the doctors (MH06, 26:716717), "to be able to get the real story or correct diagnoses you have to try and interact in a language that the patients understand". Some of the patients are persuaded at home by their family members through support and sharing of healthcare knowledge. Some are even persuaded by being advised on how to take their medications effectively and how to respond to certain medical conditions, especially those patients who are on chronic medications.

Many of the practitioners were motivated to deliver the best services, as well as to adhere to the privacy, confidentiality, and security standards of protecting the patients' healthcare information, as they had signed an ethical code of conduct. The practitioners are bound not only by an ethical code of conduct but also their employment contract to provide close care and careful observation of how they render their services. Therefore, these people are persuaded to act accordingly, within the agreed upon codes of conduct. They strive to deliver the best services to the clients who are, in this particular case, patients. According to one of the nurse participants, MH02 (2:216-218), "we do everything in compliance to the Protection of Personal Information (POPI) act were we even have to sign up a contract that abides us to this act". In order for Mukhethwa healthcare staff to deliver good services, technology is required to increase efficiency and eliminate the possibility of loss of patient data and health records. IT personnel have critical roles in making sure that support is provided to healthcare practitioners and patients whenever the need arises.

\section{Innovation Decision Process: Decision}

Many health-related decisions occur in the context of scientific uncertainty, the 'grey zone' of decision making. During the processes of diagnoses and treatments, communication becomes a vital tool in helping healthcare practitioners to understand their patients' needs, so as to make better decisions. According to MH01 (2:55-56), "I feel very bad, because it impacts on my nursing care. I cannot nurse a patient whom I don't know what the problem is". She further expressed that they need to be able to communicate to reach a consensus or agreeable decision on what needs to be done "before you help a patient as a nurse there must accurate communication" (MH01, 2:57).

However, sometimes decision poses challenges, such as delays, breaches of patient confidentiality, and a breakdown of trust from a patient who is being assisted. According to MH05 (19:590-591), "there is an ethical code of conduct that they have to adhere to, in terms of patients' confidentiality". In many areas, including the maternity section, they have already started using technology, such as a mobile application called 'mum connect'. This application is intended to improve timely access 
to emergency and general health services and information, which is ultimately, to better manage patient's care, reduce pregnancy complications, and alleviate unnecessary miscarriages. The decision to employ technology therefore needs to be supported and used by the patients. According to one of the health practitioners, MH02 (5:149-150), "patients get their technology device, and we provide the application and network connectivity".

\section{Innovation Decision Process: Implementation}

$\mathrm{MH}$ is currently implementing mobile health system in various aspects of their care, in attempts to addressing language barriers between healthcare practitioners and patients. According to one of the participants, MH01 (3:90), "every time we have a communication barrier, it becomes a problem of the hospital". This leads to the use of the system before it is fully implemented. The implementation of 'mum connect' was influenced by a large number of pregnant women who might have suffered from complications during their pregnancy that could have been prevented if only there had been a way to communicate with others about their challenges. In MH01's (5:157-159) view: "currently, the applications are developed in English, which is a problem on its own. We may need to create the applications in the indigenous languages".

The implementation of mobile health technologies within $\mathrm{MH}$ was influenced by many factors, such as language barriers between healthcare practitioners and patients, monitoring of a patient's health progress, reminders for a patient's hospital visitations, increase healthcare quality, expanding access to services, cost reductions, and improved personal wellness and public health. At the hospital, the implementation of mobile health is undeniably a challenge attributable to the broad scope of services that mobile health needs to address, such as the factors mentioned above, the lack of resources, such as infrastructure, and the very limited cost. According one of the participants, MH05 (35: 1142-1143), "we only have apps that are using English. We have to consider other languages". However, some participants still feel that this will not address the challenges per se, as the issue is not necessarily the English language but the difficulty of specific healthcare terminology, which cannot easily be translated to indigenous languages.

\section{Innovation Decision Process: Confirmation}

The use of mobile technology was not yet fully realised; some use this technology in part, while others do not even know about the existence of these technologies. Those who make use of the technology confirmed that it is working appropriately. While the short text message functionality is available, some participants struggle to use it because of language barriers. According to one of the participants, "some of the patients can't even read English but we do have a 'SMS' text message facility" (MH01, 3:77). MH sends short text messages to everyone, irrespective of whether or not they understand English. However, language is not the only issue. A lack of interaction causes difficulty in responding to the short text messages, hence some patients avoid using the technology.

The intangible benefits of going electronic include enhanced patient safety, enhanced quality of care, better provider-patient relationships and improved communication. According to one of the participants, MH06 (22:692-694), "it can save time and protect invasion of privacy because some people feel free only when they are with the healthcare practitioner alone without the presence of interpreter or family members". Finally, the widespread adoption of mobile technologies within MH will allow the achievement of system connectivity and information exchange between practitioners, healthcare facilities, and ultimately, between patients and the community. However, the realisation of these benefits depends on how MH has implemented and deployed the different mobile technologies. Thus far, the responses have been positive: "patients actually love it, and enjoying the using the technology" (MH06, 25:774-775). 


\section{FINDINGS AND DISCUSSION}

From the data analysis using ANT and DOI, as presented in the above section, six critical factors were found to influence the translation of language semantics in delivering and receiving of healthcare services at $\mathrm{MH}$. The factors were found by employing subjective reasoning, based on which a Framework (Figure 2) was developed. The factors are hospital management and structure, interactive systems, language barrier, support groups, technological know-how, and cultural and traditional affiliation. The discussion below should be read with the Framework in order to gain a better understanding of the results.

\section{Hospital Management and Structure}

The hospital has manual processes, from when the patients come in for consultation with a nurse, to when they receive medication or prescriptions. As revealed from the analysis, the manual process allows the management structure of the hospital to have complete control and power over the activities of the health practitioners and the general events of the hospital. The structure gives the management the ability to track and measure performances through transparency of all roles and responsibilities. However, the rigid management sometimes thwarted innovations attempts.

The structure and style of management affects the innovations, knowledge sharing and skills transfer, which had impact on translation of language, in that certain information or practices were not allowed to be shared freely and willingly. As a result, some of the health practitioners were short of know-how on the use of technological artefacts. Additionally, those who tried to be innovative could not share their thoughts, knowledge, or the concept they had in mind based on the management rules that guided them. This either prevents or limits some health practitioners from the use of technology and prolonged their traditional ways of doing things. Such limitations had a definite impact on how

Figure 2. Interaction between public healthcare practitioners and patients

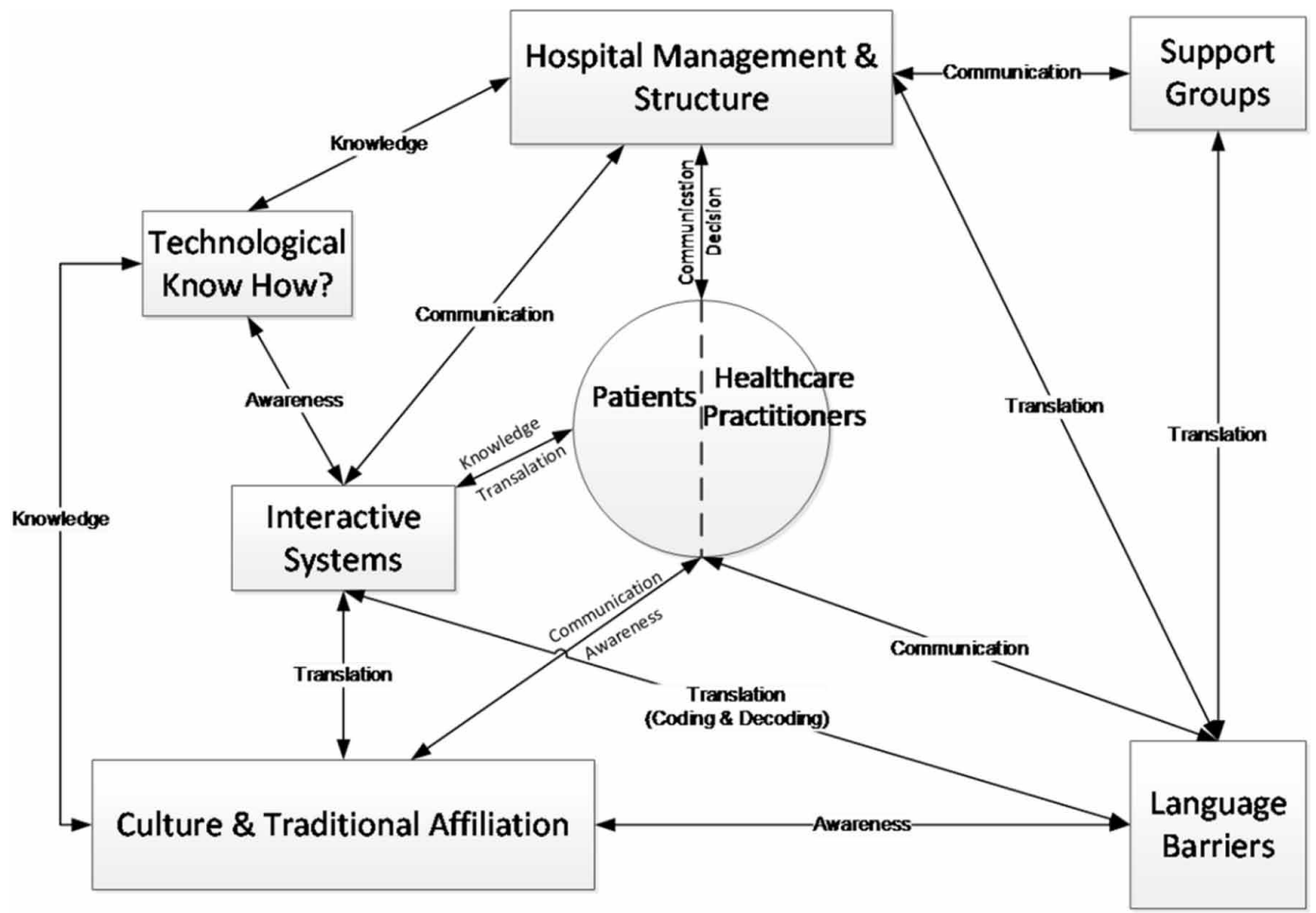


the information was translated, as the semantics around the meanings of words differs according to background and place of origin.

Another effect of the rigidity of the management structure was that the experiences and knowledge gained from practices and activities were not documented; this information stayed with the managers. Such knowledge was barely shared or communicated with those groups which were tasked with supporting the activities of the health practitioners. This causes mistake that often has consequences for the patients.

\section{Support Groups}

As revealed from the analysis, there were different groups of patients who receive support from the hospital. The groups were consciously and unconsciously formed based on health conditions and language of communication. The groups were linked to the hospital management. This was based on relationship and interaction between patients and hospital management, towards providing and receiving healthcare services. Moreover, the groups had neither relationships nor communications with each other. Even when some members of one group wanted to converse with others, the spoken languages that were used for communication were not only different, the semantics were difficult to translate.

The lack of communication between the groups and the inability of the hospital management to link the groups together occasionally led to duplication of efforts and services to the individual groups. Furthermore, some groups favoured certain health practitioners to provide service to them, primarily based on the fact that they spoke the same language, in which they felt comfortable and understood by these healthcare practitioners. Even when they were offered interpreters, they insisted on their preferred health practitioners because some of the patients did not know that the translation could be accurate owing to some semantics in their languages.

The duplication of effort that was encountered impacted how hospital management rendered their services. A process, such as capturing a patient's demographics, contains information that does not change and can be carried across. They had to do the process twice or more, generating unproductivity of practitioners and wasting of time and resources due to these non-interactive systems. The practitioners ended up spending more time doing one task over and over again, affecting their progress and wasting time that could have spent learning other skills. Therefore, the workload increased and as a result, there was no time for skills or knowledge transfer.

\section{Technological Know-How}

There were issues of technological (information systems) know-how from both patients and health practitioners' perspectives. The hospital had systems which were intended to enhance the services provided to the patients. However, many of the patients could not make use of some of these available systems. For example, some patients could not schedule appointments via self-management care systems, which allows taking daily wellness measurements, and send the results to the practitioners. Therefore, by not being able to do these, the patients had no choice but to physically visit the hospital, even as their condition was worsening. With some patients, their conditions worsened at the time they arrived at the hospital, and yet they were unable to communicate this with practitioners, resulting in delayed medical processes and procedures and inadequate diagnoses.

Additionally, some healthcare practitioners at $\mathrm{MH}$ were not familiar or did not know how to make use of some systems that were available and accessible to them. As a result, in some instances, some of those practitioners who were technically challenged referred patients to colleagues who were knowledgeable about the systems. As a result of this, they failed to document some patients' cases accurately by unknowingly omitting information. This was not a deliberate action by the health practitioners, but this was because they lacked the necessary know-how and did not know how to document patients' information using the hospital system. Such actions have consequences, impacting on the kind of prescriptions, medications, and overall services that are provided to hospital patients. 


\section{Interactive Systems}

The challenges with technological know-how made many of the employees at the hospital rely heavily on a manual system. This type of system was unilaterality and unconsciously adopted by the patients, healthcare practitioners, and pharmacists. This type of adoption - which Actor Network Theory refers to as enrolment - made things difficult, creating little or no room for diffusion of automated systems. Additionally, the longevity of the manual system meant that it was the norm, which made it difficult for interaction where different languages were spoken by both patients and healthcare providers. Thus, human translators were sometimes employed, but this often did not help as it was difficult to find those who could interpret the semantics accurately from various African languages into English. For example, in the Tshivenda language, they do not have the correct semantics or a word for 'cancer', as the natives generally refer to it as 'Tshilonda' which means 'wound' when translated literally into English.

The use of an automated interactive system could capture and translate such critical semantics once, and then continuously, without an interpreter who risks not providing the correct meaning. Moreover, an automated system could be used to protect individual privacy and enhance free communication. This was because some of the patients, particularly those who were not educated, were too shy or scared to expressed themselves on critical health conditions, such as tuberculosis and AIDS/HIV. An interactive system can enable faceless interaction, while simultaneously and importantly protecting the privacy of individuals. Such systems can be enabled and supported by mobile systems, thereby limiting or eradicating language barriers between patients and healthcare service providers.

\section{Language Barriers}

As revealed in the analysis, there was a serious language barrier between patients and health practitioners, including pharmacists, in the provision of healthcare, primarily due to the reliance on many different languages for communication and interaction for providing and receiving healthcare services. As a result of the barrier, some healthcare activities were not accurately or thoroughly documented as they could not translate some of the semantics. Therefore, gaps were left, significant enough to sometimes impact the prescriptions and dispensing of medications that patients received.

The language barrier also caused delays in carrying out services by health practitioners. For example, many of the healthcare practitioners at MH depended on their colleagues for translation when they did not understand or speak the same language as the patient to whom they were intending to provide services. As revealed from the analysis, this situation sometimes worsened when colleagues were also not fluent in the spoken language of the patients. These types of situations of misinterpretation sometimes led to wrong prescriptions. Therefore, there is a clear need for direct automatic translation for correctness, for completeness, and for accuracy. This translation can be enabled and supported by mobile systems to ensure consistency and avoid problematic repetitions. Moreover, language barriers influence divides that are already in place because of cultural and traditional affiliation, which can then also be addressed using mobile systems.

\section{Culture and Traditional Affiliation}

At $\mathrm{MH}$, there were patients who refused to interact practitioners of the opposite gender based on their cultural belief and traditional affiliations. The data, for example, showed a woman who was in a coma when she was initially brought into the hospital. She was treated and brought to stable condition. But when she realised that she was treated by a male practitioner, she became unwell and depressed, because her culture does not allow a man who is not her husband to touch her.

Most of the time, the issue is more about communication. The factors about culture and tradition are often not properly communicated to the nurses and doctors, because such patients can be treated for their health conditions without touching them. But the semantics in the way it is said, comes across differently such that it is almost an abomination, though it is not. It is almost saying that certain parts 
of a woman cannot be touched by another male. However, this part of the process is missing and needs to be addressed. Furthermore, proper communication can allow an appropriate arrangement in anticipation of receiving patients who are guided by culture and tradition.

\section{CONCLUSION}

In South African, although there are eleven official languages, English language is commonly used as medium of communication. This is despite the fact that majority of healthcare recipients cannot speak the English language fluently or do not understand it coherently. Thus, this study proposed a solution through its framework, to addressing this difficulty in each facility, for health practitioners who comes from other part of the country, or outside of the country, in their quest to providing services. Thus, efforts has been made to employ ICT solutions, to bridge the communication gap between the providers and recipients of healthcare in the country, which has not been successful.

The contributions of the study are two-fold, namely, practically and methodologically. Practically, the contribution of the study comes from the framework (Figure 3) that was developed. In practice, both healthcare practitioners and ICT specialists can employ the factors identified in the framework as standard guidelines. This can be done in practice by formulating criteria for each of the factors. The criteria can be used to guide the selection of ICT Solutions in ensuring appropriateness in addressing language barrier between service providers and recipients. This helps reduce challenges in the translation of language in the South African healthcare facilities. Methodologically, the combined use of the two sociotechnical theories, ANT and DOI as lenses to guide the analysis of the data has been the primary methodological contribution of the study. To our knowledge, as at the time of this study, both theories have not been complementarily used in any IS research. From this viewpoint, the complementary use of the theories make a significant contribution to the field of IS research through its addition to existing literature. 


\section{REFERENCES}

Aizstrauta, D., Ginters, E., \& Eroles, M. A. P. (2015). Applying theory of diffusion of innovations to evaluate technology acceptance and sustainability. Procedia Computer Science, 43, 69-77. doi:10.1016/j.procs.2014.12.010

Anari, S. M., \& Sanjarani, A. (2016). Application of Baker's Model in Translating Quran-Specific Cultural Items. Journal of Language Sciences \& Linguistics, 4(3), 145-151.

Ayong, Q., \& Atanga, J. (2017). Overcoming Nursing Care Challenges in a Multicultural Health Care Setting. Retrieved from https://www.theseus.fi/bitstream/handle/10024/138148

Baker, D. W., Hayes, R., \& Fortier, J. P. (1998). Interpreter use and satisfaction with interpersonal aspects of care for Spanish-speaking patients. Medical Care, 36(10), 1461-1470. doi:10.1097/00005650-199810000-00004 PMID:9794340

Ball, R. D., Bertone, V., Carrazza, S., Deans, C. S., Del Debbio, L., Forte, S., \& Ubiali, M. et al. (2015). Parton distributions for the LHC Run II. Journal of High Energy Physics, 2015(4), 40. doi:10.1007/JHEP04(2015)040

Barr, N., Vania, D., Randall, G., \& Mulvale, G. (2017). Impact of information and communication technology on interprofessional collaboration for chronic disease management: A systematic review. Journal of Health Services Research \& Policy, 22(4), 250-257. doi:10.1177/1355819617714292 PMID:28587494

Baškarada, S. (2014). Qualitative case study guidelines. Baškarada, S.(2014). Qualitative case studies guidelines. Qualitative Report, 19(40), 1-25.

Brown, D. W. (2018). U.S. Patent No. 9,875,023. Washington, DC: U.S. Patent and Trademark Office.

Burns, L. R. (Ed.). (2012). The business of healthcare innovation. Cambridge University Press. doi:10.1017/ CBO9781139176620

Callon, M. (1986). Some elements of a sociology of translation: domestication of the scallops and the fishermen of St Brieuc Bay. In J. Law (Ed.), Power, Action \& Belief: A New Sociology of Knowledge? (pp. 196-229). London: Routledge.

Carston, R. (2013). 12. Implicature, Explicature, and Truth-Theoretic Semantics. The Semantics-Pragmatics Boundary in Philosophy, 261.

Chomsky, N. (1975). Reflections on language. New York: Pantheon. ChomskyReflections on language 1975.

Dong, T., \& Cremers, A. B. (2014, April). A novel machine translation method for learning Chinese as a foreign language. Proceedings of the International Conference on Intelligent Text Processing and Computational Linguistics (pp. 343-354). Springer. doi:10.1007/978-3-642-54903-8_29

Farooq, M. B., \& De Villiers, C. (2017). Telephonic qualitative research interviews: When to consider them and how to do them. Meditari Accountancy Research, 25(2), 291-316. doi:10.1108/MEDAR-10-2016-0083

Gandhi, J. K., Burstin, H. R., Cook, E. F., Puopolo, A. L., Haas, J. S., Brennan, T. A., \& Bates, D. W. (1998). Drug Complications in Outpatients. Journal of General Internal Medicine, 15(3), 149-154. doi:10.1046/j.15251497.2000.04199.x PMID:10718894

Gany, F., Kapelusznik, L., Prakash, K., Gonzalez, J., Orta, L. Y., Tseng, C. H., \& Changrani, J. (2007). The impact of medical interpretation method on time and errors. Journal of General Internal Medicine, 22(2), 319-323. doi:10.1007/s11606-007-0361-7 PMID:17957418

Grove, S. K., Burns, N., \& Gray, J. R. (2014). Understanding nursing research: Building an evidence-based practice. Elsevier Health Sciences.

Idalovichi, I. B. Y. (2014). Symbolic Forms as the Metaphysical Groundwork of the Organon of the Cultural Sciences (Vol. 1). Cambridge Scholars Publishing.

Iftikhar, S., Ishaq, A. M., Ahmad, H. F., \& Fatima, K. (2010, October). Introducing semantics in DHTs for Grid services in a semantic registry. Proceedings of the 20106 th International Conference on Emerging Technologies (ICET) (pp. 382-387). IEEE. doi:10.1109/ICET.2010.5638457

Iyamu, T. (2015). Application of underpinning theories in information systems. Heidelberg Press. 
Iyamu, T., \& Tatnall, A. (2009). An actor-network analysis of a case of development and implementation of IT strategy. International Journal of Actor-Network Theory and Technological Innovation, 1(4), 35-52. doi:10.4018/ jantti.2009062303

Kohn, L. T., Corrigan, J. M., \& Donaldson, M. S. (Eds.). (1999). To Err is Human: Building a Safer Health System. Washington, D.C.: National Academy Press.

Munday, P. (2016). The case for using DUOLINGO as part of the language classroom experience. RIED: revista iberoamericana de educación a distancia, 19(1), 83-101.

Naidoo, S. (2014). Transcultural and language barriers to patient care. South African Dental Journal, 69(9), 425-425. PMID:26571928

Nemutanzhela, P., \& Iyamu, T. (2016). Introducing mobile device for health services: The semantics of language translation. In Maximizing healthcare delivery and management through technology integration (pp. 101-112). Hershey, PA: IGI Global. doi:10.4018/978-1-4666-9446-0.ch007

Rogers, E. M. (2003). Diffusion of Innovations (5th ed.). New York, NY: Free Press.

Rodger, J. A. (2015). Discovery of medical Big Data analytics: Improving the prediction of traumatic brain injury survival rates by data mining Patient Informatics Processing Software Hybrid Hadoop Hive. Informatics in Medicine Unlocked, 1, 17-26. doi:10.1016/j.imu.2016.01.002

Rouleau, G., Gagnon, M. P., \& Côté, J. (2015). Impacts of information and communication technologies on nursing care: An overview of systematic reviews (protocol). Systematic Reviews, 4(1), 75. doi:10.1186/s13643015-0062-y PMID:26002726

Saleem, J. J., Flanagan, M. E., Wilck, N. R., Demetriades, J., \& Doebbeling, B. N. (2013). The next-generation electronic health record: Perspectives of key leaders from the US Department of Veterans Affairs. Journal of the American Medical Informatics Association, 20(e1), e175-e177.

Scott, S., \& McGuire, J. (2017). Using Diffusion of Innovation Theory to Promote Universally Designed College Instruction. International Journal on Teaching and Learning in Higher Education, 29(1), 119-128.

Tatnall, A. (Ed.). (2012). Social and professional applications of actor-network theory for technology development. IGI Global.

Vermeulen, R., Portengen, L., Silverman, D. T., Garshick, E., \& Steenland, K. (2014). Meta-Analysis of Lung Cancer Risk from Exposure to Diesel Exhaust: Vermeulen et al. Respond. Environmental Health Perspectives, 122(9), A230-A231. doi:10.1289/ehp.1408482R PMID:25181539

Walsham, G. (1997). Actor-network theory and IS research: current status and future prospects. In Information systems and qualitative research (pp. 466-480). Boston, MA: Springer. doi:10.1007/978-0-387-35309-8_23

Woloshin, S., Bickell, N. A., Schwartz, L. M., Gany, F., \& Welch, H. G. (1995). Language barriers in medicine in the United States. Journal of the American Medical Association, 273(9), 724-728. doi:10.1001/ jama.1995.03520330054037 PMID:7853631

Yagos, W. O., Olok, G. T., \& Ovuga, E. (2017). Use of information and communication technology and retention of health workers in rural post-war conflict Northern Uganda: Findings from a qualitative study. BMC Medical Informatics and Decision Making, 17(1), 6. doi:10.1186/s12911-016-0403-3 PMID:28068980

Yin, R. K. (2013). Validity and generalization in future case study evaluations. Evaluation, 19(3), 321-332. doi:10.1177/1356389013497081

Zakaria, N., Affendi, S., \& Zakaria, N. (2010). Managing ICT in healthcare organization: culture, challenges, and issues of technology adoption and implementation. In Health Information Systems: Concepts, Methodologies, Tools, and Applications (pp. 1357-1372). Hershey, PA: IGI Global. 
Dr Phathutshedzo Makovhololo has fourteen years work experience in Information Technology (IT). Her areas of expertise include Business analysis, Business Intelligence, Project management, and solution architecture. From training and academic front, she has published many research articles in Journals.. Dr Makovhololo holds a PhD in Informatics from Cape Peninsula University of Technology; and Maters' and BTech degrees from Tshwane University of Technology, Pretoria. Dr. Makovhololo is an author of numerous peer-reviewed journal and conference proceeding articles. Her research interests include Competitive Intelligence, Mobile Health, Information Systems, language semantics and focuses on Diffusion of Innovation theory (Dol) and now also exploring Actor Network theory (ANT) and other different theories.

Tiko Iyamu holds a PhD in Information Systems. He is currently a Research Professor at the faculty of Informatics and Design, Cape Peninsula University of Technology, Cape Town, South Africa. He was previously with the Tshwane University of Technology, South Africa and the Namibia University of Science and Technology, Windhoek, Namibia. Prior to his fulltime appointment in academic in 2009, Professor lyamu held several positions in both Public and Private Institutions in South Africa. Professor lyamu has authored books, and research articles in book chapters, journals and conference proceedings. 